\title{
Windowless microfluidic platform based on capillary burst valves for high intensity $x$ - ray measurements
}

Vig, Asger Laurberg; Haldrup, Kristoffer; Enevoldsen, Nikolaj Brandt; Thilsted, Anil Haraksingh; Eriksen, Johan; Kristensen, Anders; Feidenhans'I, Robert Krarup; Nielsen, Martin Meedom

Published in:

Review of Scientific Instruments

Link to article, DOI:

$10.1063 / 1.3262498$

Publication date:

2009

Document Version

Publisher's PDF, also known as Version of record

Link back to DTU Orbit

Citation (APA):

Vig, A. L., Haldrup, K., Enevoldsen, N. B., Thilsted, A. H., Eriksen, J., Kristensen, A., Feidenhans'l, R. K., \& Nielsen, M. M. (2009). Windowless microfluidic platform based on capillary burst valves for high intensity $\mathrm{x}$-ray measurements. Review of Scientific Instruments, 80(11), 115114. https://doi.org/10.1063/1.3262498

\section{General rights}

Copyright and moral rights for the publications made accessible in the public portal are retained by the authors and/or other copyright owners and it is a condition of accessing publications that users recognise and abide by the legal requirements associated with these rights.

- Users may download and print one copy of any publication from the public portal for the purpose of private study or research.

- You may not further distribute the material or use it for any profit-making activity or commercial gain

- You may freely distribute the URL identifying the publication in the public portal 


\title{
Windowless microfluidic platform based on capillary burst valves for high intensity $\mathrm{x}$-ray measurements
}

\author{
Asger Laurberg Vig, ${ }^{1}$ Kristoffer Haldrup, ${ }^{2}$ Nikolaj Enevoldsen, ${ }^{1}$ Anil Haraksingh Thilsted, ${ }^{1}$ \\ Johan Eriksen, ${ }^{1}$ Anders Kristensen, ${ }^{1, a)}$ Robert Feidenhans'l, ${ }^{2}$ and \\ Martin Meedom Nielsen ${ }^{2}$ \\ ${ }^{1}$ Department of Micro and Nanotechnology, DTU Nanotech, Technical University of Denmark, \\ Building 345east, Ørsteds Plads, DK-2800 Kongens Lyngby, Denmark \\ ${ }^{2}$ Centre for Molecular Movies, Niels Bohr Institute, University of Copenhagen, Universitetsparken 5, \\ DK-2100 Copenhagen East, Denmark
}

(Received 7 September 2009; accepted 16 October 2009; published online 25 November 2009)

\begin{abstract}
We propose and describe a microfluidic system for high intensity x-ray measurements. The required open access to a microfluidic channel is provided by an out-of-plane capillary burst valve (CBV). The functionality of the out-of-plane CBV is characterized with respect to the diameter of the windowless access hole, ranging from 10 to $130 \mu \mathrm{m}$. Maximum driving pressures from 22 to 280 mbar corresponding to refresh rates of the exposed sample from $300 \mathrm{~Hz}$ to $54 \mathrm{kHz}$ is demonstrated. The microfluidic system is tested at beamline ID09b at the ESRF synchrotron radiation facility in Grenoble, and x-ray scattering measurements are shown to be feasible and to require only very limited amounts of sample, $<1 \mathrm{ml} / \mathrm{h}$ of measurements without recapturing of sample. With small adjustments of the present chip design, scattering angles up to $30^{\circ}$ can be achieved without shadowing effects and integration on-chip mixing and spectroscopy appears straightforward.

(C) 2009 American Institute of Physics. [doi:10.1063/1.3262498]
\end{abstract}

\section{INTRODUCTION}

The past decade has seen considerable advances within the time-resolved synchrotron-based studies of chemistry and biology in solution, spanning from fundamental chemistry on the $100 \mathrm{ps}$ time scale to conformation changes in solvated proteins on the microsecond scale. ${ }^{1}$ In both of these time regimes, the arrival of the new x-ray free electron laser (XFEL) sources is foreseen to greatly increase the amount of information that can be extracted from liquid-state experiments due to the exceptional brilliance, time resolution, and coherence properties. ${ }^{2}$

In order to take full advantage of the possibilities offered by the new x-ray sources, some crucial points in sample handling will have to be addressed to preserve coherence, subpicosecond time resolution, and to prevent sample damage from the extremely bright $\mathrm{x}$-ray pulse as the $\mathrm{x}$-ray figure-of-merit, brilliance (brilliance is defined as photons $/ \mathrm{s} / \mathrm{mrad}^{2} \mathrm{~mm}^{2} 0.1 \%$ bandwidth), increases by ten orders of magnitude over current synchrotron sources. To prevent sample damage and debris buildup, the liquid sample should ideally be replaced between every x-ray pulse, which will come at $120 \mathrm{~Hz}$ at the Linac Coherent Light Source (LCLS) at Stanford and at $10 \mathrm{~Hz} / 5 \mathrm{MHz}$ at the European XFEL. Preserving the pulse-length limited time resolution dictates that the sample must be thin due to the difference in light speed between the UV-VIS-infrared laser pump pulse and the $\mathrm{x}$-ray probe pulse as they propagate through the sample. The accumulated time difference $\Delta t$ between the two pulses is given by $\Delta t=l / c(1-n)$, with $l$ the path length, $c$ the speed of light, and $n$ the refractive index of the media at a

\footnotetext{
${ }^{a)}$ Electronic mail: anders.kristensen@nanotech.dtu.dk.
}

given pump pulse wavelength. Taking water $(n=1.33)$ as a typical example, a path length of $100 \mu \mathrm{m}$ corresponds to a time delay between the pulses slightly larger than 100 fs thus limiting the sample thickness to $50-100 \mu \mathrm{m}$ if $100 \mathrm{fs}$ time resolutions is to be conserved in the case of copropagating pump and probe beams. ${ }^{3}$ Such sample dimensions are routinely achieved in microjet systems ${ }^{4,5}$ and droplet shooters, ${ }^{6}$ however, the curved interfaces inherent in these systems may cause refraction effects as well as decrease the coherence properties of the x-ray beam. Free-flowing films ${ }^{7}$ with planar interfaces perpendicular to the $\mathrm{x}$-ray beam may also reach the required maximum thickness but as well as stability, lack of vacuum compatibility, and large sample volumes are known issues. Finally, cuvette-based systems ${ }^{8}$ might not be able to withstand the x-ray beam due to the significant energy deposited by absorption.

Taking all of the points above into consideration, an ideal sample system for XFEL measurements on liquid systems will have a thin (sub-75 $\mu \mathrm{m}$ ) and stable sheet of liquid flowing perpendicular to the $\mathrm{x}$-ray beam. The flow velocity of the sample should furthermore be sufficient to reach a refresh rate of the exposed volume exceeding the repetition frequency of the x-ray pulses $(10 \mathrm{~Hz} / 120 \mathrm{~Hz} / 5 \mathrm{MHz})$ while also ensuring that no material other than the sample should be in the X-ray beam path. In the following, we present a windowless, microfluidic sample chip based on an out-ofplane implementation of the capillary burst valve (CBV) principle that meets these demands. The proposed design also enables straightforward integration of other lab-on-achip applications, such as waveguides, lasers, ${ }^{9}$ mixers, ${ }^{10}$ and separators. ${ }^{11}$

The functionality of the windowless $\mathrm{x}$-ray access hole in the proposed microfluidic platform is based on a CBV, sug- 


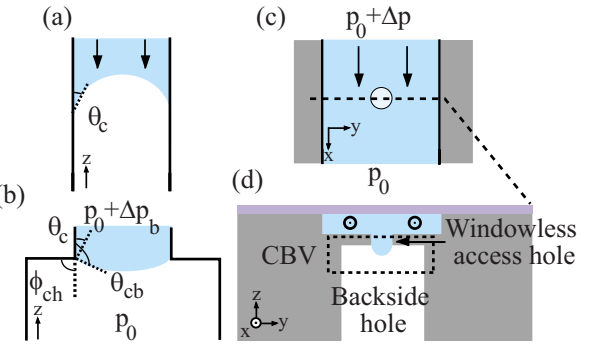

FIG. 1. (Color online) (a) Contact angle $\theta_{c}$ of a liquid propagating due to capillary forces in a hydrophilic channel. (b) A CBV at the bursting point, exposed to the burst pressure $\Delta p_{b}$. [(c) and (d)] Schematic layout of the proposed windowless microfluidic platform as seen from above (c) and in cross-sectional view (d). It consists of a CBV placed in the middle of a flow channel. The dashed box in (d) refers to the situation depicted in (b).

gested and tested earlier. ${ }^{12,13} \mathrm{CBV}$ s rely on the surface tension of the liquid at a liquid/solid/gas interface and it consists of a narrow channel with a sudden widening at a given point along its length - the following description represents liquid in a hydrophilic channel. When a liquid reservoir is brought into contact with the narrow channel, the liquid is dragged into the channel by capillary forces. The liquid propagates with a characteristic contact angle, the angle between the channel wall, and the liquid front $\theta_{c}$, see Fig. 1(a). This angle is identical to the contact angle of a drop of the same liquid on a flat horizontal surface. If the size of the channel abruptly increases with an angle $\phi_{\mathrm{ch}}$, the liquid stops propagating due to the cost in energy associated with the significant increase in surface area. Additional energy in form of an increase in the pressure has to be applied at the entrance of the narrow channel to restore the contact angle with respect to the surface connecting the narrow and wide channel segment, $\theta_{\mathrm{cb}}$ $=\theta_{c}+\phi_{\mathrm{ch}}$, necessary for the liquid to continue along the wall into the wider channel segment. Figure 1(b) shows the situation just before the valve will burst. The central performance measure of a CBV is the burst pressure $\Delta p_{b}$ and in previous work ${ }^{12-15}$ the burst pressure has been predicted and confirmed to be proportional to the liquid surface tension $\gamma$ and inversely proportional with the channel dimension $D$. The bursting pressure for a hydrophilic or hydrophobic channel with circular cross section can be derived from the Young-Laplace equation, and is given by Eq. (1).

$$
\Delta p_{b}=\frac{-4 \gamma \cos \theta_{\mathrm{cb}}}{D}
$$

In previous demonstrations, the $\mathrm{CBV}$ has been placed in the plane of the microfluidic network, e.g., for use in microfluidic centrifugal systems. ${ }^{16-18}$ Employing a CBV perpendicular to the direction of the sample has not been implemented previously and in the following it will be described how this enables windowless microfluidic measuring platforms.

The microfluidic chip presented in this work has channels in the surface of the chip and a perpendicular windowless access hole through the bulk, as illustrated in Figs. 1(c) and $1(\mathrm{~d})$. (a)

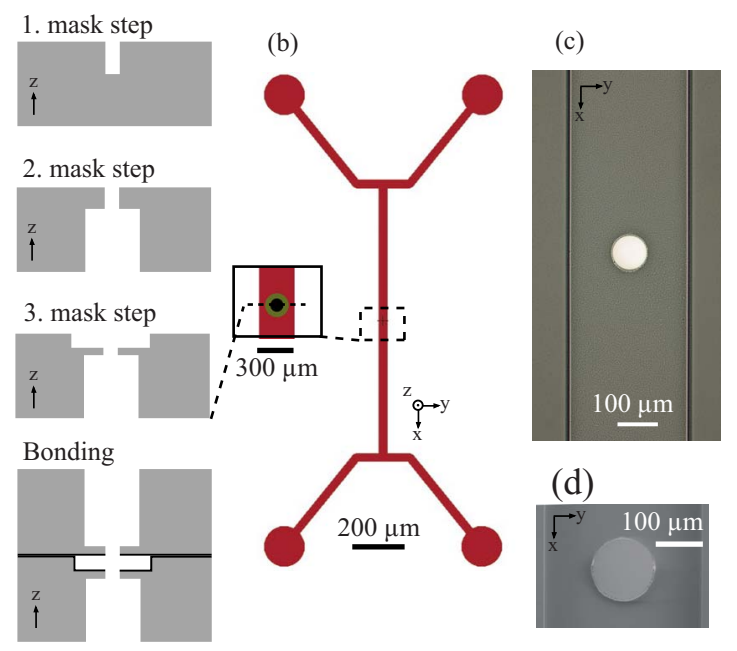

FIG. 2. (Color online) (a) Simplified outline of the fabrication process. Three mask steps are involved: (1) the windowless beam access, (2) the backside holes in the CBV, and (3) the microchannel structure. Finally, fusion bonding is used to seal off the channel structures. (b) Chip layout with a closeup of the CBV region. The outer (shaded) circle corresponds to the backside hole and the inner (black) circle is the windowless x-ray beam access hole. (c) Microscope image with backside illumination, showing a finished device before bonding. The hole is $111 \mu \mathrm{m}$ in diameter. (d) SEM image of the same device, centered on the CBV.

\section{DESIGN AND FABRICATION OF THE WINDOWLESS MICROFLUIDIC DEVICE}

As Fig. 2 illustrates, the microfluidic sample cell is designed with two inlet channels merging into a $1-\mathrm{cm}$-long straight channel which terminates in two outlet channels identical to the inlets. All channels are $300 \mu \mathrm{m}$ wide and $78 \mu \mathrm{m}$ deep. In the middle of the straight channel the CBV is positioned. Figure 2(b) illustrates a top view of the device layout with a closeup of the CBV region in the inset. The large circle indicates the backside hole and the small circle indicates the windowless access hole. The diameter of the large backside hole of the CBV is kept constant at $200 \mu \mathrm{m}$ but can be arbitrarily increased for better beam access. The diameter of the window itself is varied from 10 to $130 \mu \mathrm{m}$ and the thickness of the window membrane is $25 \mu \mathrm{m}$ in the substrate wafer and $40 \mu \mathrm{m}$ in the lid wafer. For x-ray measurements the thickness of the window membrane should be minimized as much as possible as stagnant fluid in this volume will not be exchanged and may give rise to spurious background signals as discussed in further detail below.

The microfluidic devices are fabricated using planar silicon $(\mathrm{Si})$ microfabrication techniques. $500 \mu \mathrm{m}$ thick, double polished, single crystalline $\langle 100\rangle \mathrm{Si}$ wafers are used. The fabrication consists of three masking steps. Each step employs a deep reactive ion etch (DRIE) utilizing the Bosh process,${ }^{19}$ thereby transferring the structures anisotropically into the Si wafer. All masking steps are illustrated in Fig. 2(a). In each of the three steps, I-line UV contact lithography is used to transfer the mask layout into an 10- $\mu$ m-thick UV sensitive resist layer (AZ4562 standard resist), on top of the $\mathrm{Si}$ wafer. The resist functions as an etch mask in the subsequent DRIE process optimized for deep etching [etch: $\mathrm{SF}_{6}: 230 \mathrm{SCCM}$ (SCCM denotes cubic centimeter per 
minute at STP), $\mathrm{O}_{2}: 23$ SCCM, rf coil: $2800 \mathrm{~W}$, rf platen: 19 $\mathrm{W}$, cycle time: 8 s. Passivation: $\mathrm{C}_{4} \mathrm{~F}_{4}: 120 \mathrm{SCCM}$, rf coil: $1000 \mathrm{~W}$, rf platen: $0 \mathrm{~W}$, cycle time: $5 \mathrm{~s}$.). The window and the backside hole are fabricated in the two first masking steps, and in the case of the substrate wafer, the channel structures are subsequently fabricated in a third masking step. Afterwards, a $110 \mathrm{~nm}$ silicon dioxide $\left[\mathrm{SiO}_{2}\right.$, black in Fig. 2(a)] layer is thermally grown (dry oxidation, $1100{ }^{\circ} \mathrm{C}$, $50 \mathrm{~min}$ ) on both the substrate and lid $\mathrm{Si}$ wafer to prepare the wafers for fusion bonding and to make the channels more hydrophilic. Finally, after removing the $\mathrm{SiO}_{2}$ on the backside of the wafers and inside the $\mathrm{CBV}$, fusion bonding ${ }^{20}$ is used to seal the channels in the structured Si substrate wafer either with a borofloat glass wafer or a $\mathrm{Si}$ wafer with backside and access holes predefined according to the process described above. The transparent borofloat glass lid is used throughout the CBV characterization and the Si lid is used for the x-ray measurements. An example of a finished device before lid bonding is shown in Fig. 2(c) with a closeup of the CBV region in Fig. 2(d).

\section{EXPERIMENTAL RESULTS AND DISCUSSION}

\section{A. Experimental result of the CBV}

Characterization of the $\mathrm{CBV}$ was divided into three parts. The first part was an extensive investigation of the relation between the diameter of the windowless access hole and the burst pressure. A test liquid consisting of $1 \mu \mathrm{m}$ diameter fluorescent labeled polystyrene spheres dispersed in de-ionized water at a $0.0001 \mathrm{wt} \%$ solid concentration was used. The microspheres were used as marker particles to monitor the fluid flow velocity. No effect of the microspheres on the bursting pressure was observed at this concentration of microspheres nor at the highest concentration tested, $0.1 \mathrm{wt} \%$. The second part of the characterization of the CBV-based devices involved testing the functionality with two samples, relevant for ultrafast time-resolved analysis of chemical and biological species in liquid solution; $8 \mathrm{mM}$ of the highly photoactive platinum compound Tetrakis- $\mu$-pyrophosphitodiplatinate(II) (PtPOP) in $\mathrm{H}_{2} \mathrm{O}$ (Ref. 21) and 2, 5, and $10 \mathrm{mM}$ of the well-studied protein Cytochrome-C (Ref. 22) in a dedicated buffer $(50 \mathrm{mM}$ Hepes and $150 \mathrm{mM} \mathrm{NaCl}$ in de-ionized water). The third and final part of the characterization was a vacuum compatibility test to confirm that the device would work in environments below atmospheric pressure.

In all cases $500 \mu \mathrm{l}$ of the sample was added to each of the two inlets and capillary forces dragged the sample into the device. Subsequently, an overpressure, supplied from a minidiaphragm vacuum pump (VP 86 with EU-plug, VWR and Bie \& Berntsen) and controlled with a multiturn needle valve (EW-06393-60, Cole Parmer), of 3 mbar was applied to the inlets and the established flow was allowed to continue for $15 \mathrm{~min}$ in order to secure stability of device and flow. The pressure at the inlets was then increased slowly from $3 \mathrm{mbar}$ until the CBV was observed to burst. For each valve diameter, five to nine chips from the same wafer were tested to provide sufficient statistics on the reproducibility of the results presented in Fig. 3. This figure shows the measured

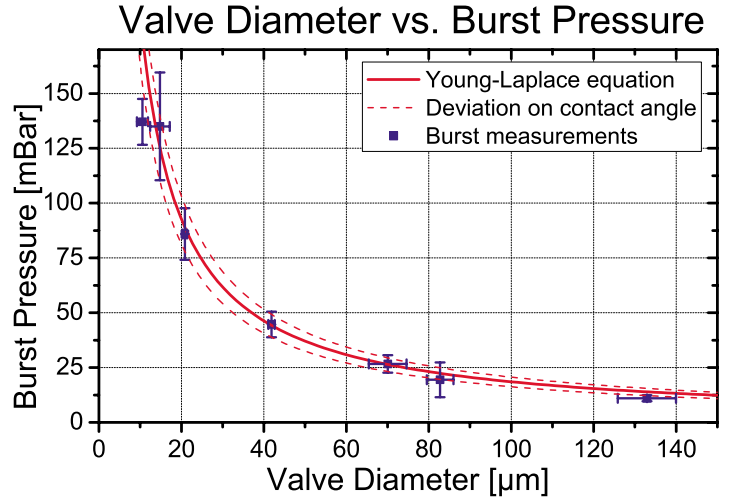

FIG. 3. (Color online) Measured (blue data points) and predicted values (red lines) of the bursting pressure as a function of the diameter of the windowless access hole in the CBV. The length of the error bars on the data points equals two standard deviations, the dashed lines denote the upper and lower predicted limits, taking fabrication imperfections into account (see text for details).

burst pressure as function of valve diameter along with the corresponding theoretical values calculated from Eq. (1). After the initial burst events reported in Fig. 3, the burst pressure was observed to depend critically on cleaning but was generally lower than the initial value. We assign this lowering of burst pressure to the presence of microspheres and other solid contaminants at the transition between the narrow and the wide part of the CBV.

The surface tension in Eq. (1) is set to the value of water on $\mathrm{Si}$ at $20^{\circ} \mathrm{C}, 72.9 \mathrm{~mJ} / \mathrm{m}^{2}$. The contact angle $\theta_{\mathrm{cb}}=\theta_{c}$ $+\phi_{\mathrm{cb}}$ was observed to depend on hole diameter due to the DRIE process of the backside hole. $\theta_{c}$ was measured (Krüss DSA100) to be $40.5^{\circ}$ with a standard deviation of $5.7^{\circ}$. The corresponding uncertainty in predicted burst pressure is represented in Fig. 3 by the dashed lines. $\phi_{\mathrm{cb}}$ as determined by scanning electron micrograph (SEM) investigations of split CBVs was determined to depend directly on the hole diameter and to range from $70^{\circ}$ for the largest access holes to $90^{\circ}$ for the smallest holes. Altogether, $\theta_{\mathrm{cb}}$ ranges from $112^{\circ}$ to $132^{\circ}$ in the devices tested in this study, and the burst pressures predicted through Eq. (1) and using these values are indicated by the solid line in Fig. 3 .

From Fig. 3, it is evident that the bursting pressure as a function of valve diameter is well described by Eq. (1). From the characterization measurements on the CBV devices, the average flow velocity in the channel was observed to depend linearly on the pressure difference between inlet and outlet as expected for a Poiseuille flow. ${ }^{23}$ The increase in average velocity per mbar pressure increase was found to be $2 \mathrm{~mm} /$ (s mbar). The maximum average velocity thus ranged from $44 \mathrm{~mm} / \mathrm{s}$ for the $130 \mu \mathrm{m}$ access holes to $540 \mathrm{~mm} / \mathrm{s}$ for the $10 \mu \mathrm{m}$ access holes corresponding to a refresh rate of the measuring volume of 0.3 and $54 \mathrm{kHz}$, respectively.

It should be noted that the angle of the air-liquid interface of the CBV is strongly dependent on the driving pressure, going from convex to concave with increasing pressure. For applications where refraction effects are of importance, the overpressure can thus be chosen to produce a flat meniscus interface.

In the compatibility test with PtPOP and Cytochrome-C 
(a)

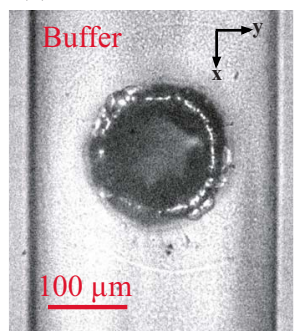

(c)

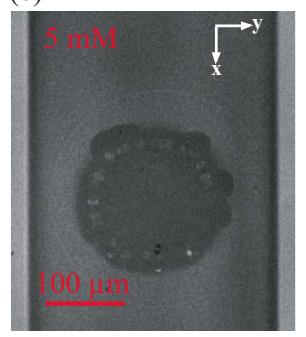

(b)

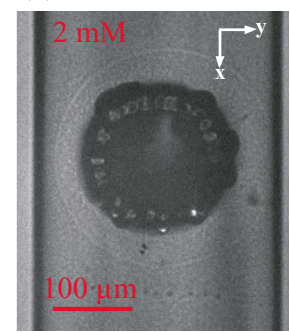

(d)

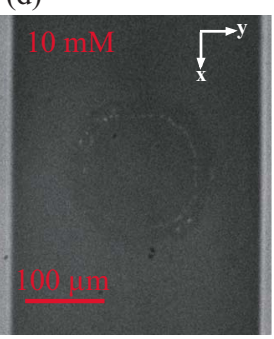

FIG. 4. (Color online) Microscope images of the microchannel at the CBV with three different concentrations of the protein Cytochrome-C: (b) $2 \mathrm{mM}$, (c) $5 \mathrm{mM}$, and (d) $10 \mathrm{mM}$ compared with only buffer (a). The buffer in these measurements is 50 and $150 \mathrm{mM} \mathrm{NaCl}$ in de-ionized water. The intensity scale of the images is equalized, so the darkness in the channel indicates the concentration, with dark corresponding to high concentration. It is evident from the images that there is no buildup of proteins in the channel segments.

the CBV did not deviate from the tests with 0.0001 wt $\%$ polystyrene microspheres in water presented in Fig. 3. These tests were carried out in an identical fashion but additionally the $500 \mu \mathrm{l}$ sample from each inlet was collected after passing the chip and from microscope inspections it was verified that it was not absorbed on the inner surfaces of the microfluidic channels. Examples of 2, 5, and $10 \mathrm{mM}$ Cytochrome-C samples in the chip are shown in Figs. 4(b)-4(d), respectively. With respect to chemical stability, the surface material of the channels, $\mathrm{SiO}_{2}$, is very stable toward biological samples as well as aggressive solvents such as those typically used for nanomaterial synthesis. The current chip design and material choice can therefore be used for most biology and synthesis studies.

Basic vacuum functionality was confirmed using a purpose-built vacuum chamber with liquid connections to the chip. The chip was tested down to chamber pressure 100 mbar with de-ionized water as the test liquid. The results were similar to those presented in Fig. 3.

\section{B. Experimental result of the x-ray measurements}

To test the proposed sample handling system under realistic operating conditions, a CBV chip with $\mathrm{Si}$ lid was mounted at the time-resolved beamline ID09b ${ }^{24}$ at the ESRF synchrotron radiation facility in Grenoble using a purposebuilt sample chuck with incorporated channels for sample supply to the chip. After preparing the system with a sample solution (water) and connecting it to the flow control system, the access hole on the chip was manually aligned to the incoming laser and $\mathrm{x}$-ray beams using a microscope. The position was subsequently refined using a point detector situated behind (downstream) the chip+holder.

Figure 5 shows the scattering pattern acquired on an area detector (Frelon) placed $10 \mathrm{~cm}$ downstream from the laser/

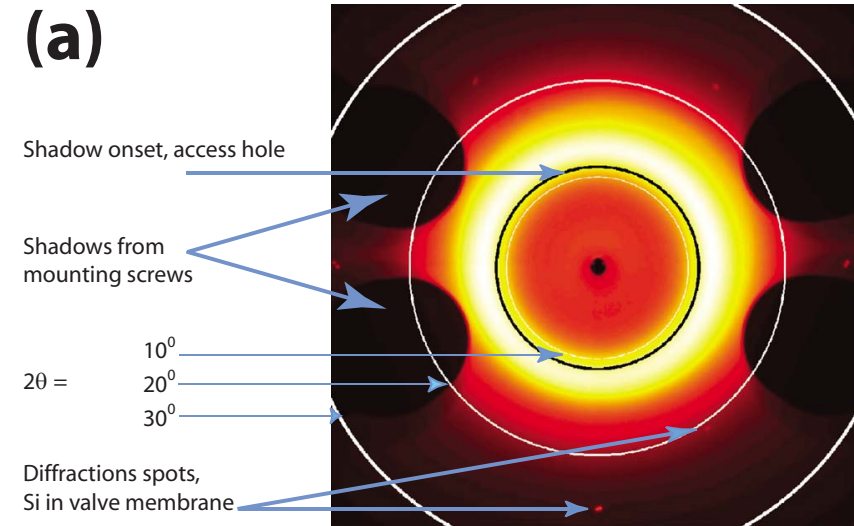

(b)

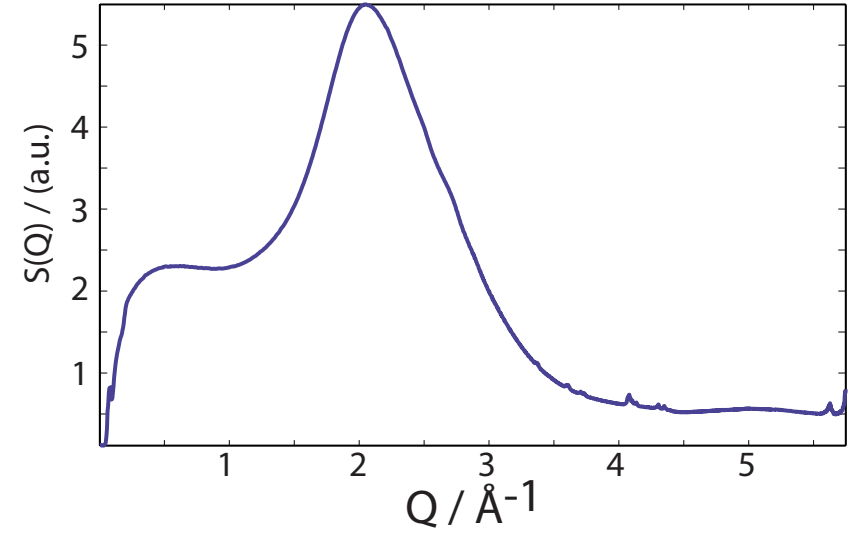

FIG. 5. (Color online) (a) Scattering pattern from a de-ionized water sample in a sample chip with a $110 \mu \mathrm{m}$ CBV. The scattering pattern was acquired over a $1 \mathrm{~s}$ period, corresponding to 986 individual photon bunches from the synchrotron, each containing $\sim 10^{9}$ photons. The blue circles in the figure denotes values of the scattering vector/scattering angles and the red circles indicate the onset of shadowing effects for a scatterer situated in the middle of the flow channel. (b) Azimuthal integration of the detector image in (a), the water peak centered at $Q=2 \AA^{-1}$ is very evident.

$\mathrm{x}$-ray overlap point positioned in the middle of the flow channel. Also shown in this figure are rings denoting values of the scattering angle $2 \theta$. Due to the narrow geometry chosen for the backside hole in the CBV, part of the Si substrate will shadow some parts of the detector. The green, dashed circle denote the point where shadowing due to absorption in $\mathrm{Si}$ exceeds $5 \%$ at $18 \mathrm{keV}$, assuming scattering from a point in the center of the channel. It is observed that only the part of the scattering pattern extending up to $2 \theta=12^{\circ}[Q$ $\left.=1.8 \AA^{-1}, Q=4 \pi / \lambda \sin \theta(\lambda \sim 0.7 \AA)\right]$ is completely unperturbed by shadowing effects with the present chip geometry. The driving pressure was kept at $3 \mathrm{mbar}$, corresponding to a flow speed of $6 \mathrm{~mm} / \mathrm{s}$ (refresh rate $=41 \mathrm{~Hz}$ ). This corresponds to a sample consumption rate of $0.5 \mathrm{ml} / \mathrm{h}$, in good correspondence with observations during the experiments.

Attempts at acquiring a time-resolved signal with a solution of TIPtPOP (Ref. 25) were not conclusive, as irradiation of the stacked/stagnant volume caused significant precipitation of Pt crystallites as well as strong perturbations of the scattering signals due to local heating. However, transient heating of the precipitated crystallites was observed on the subnanosecond time scale, confirming that spatial overlap between laser and $\mathrm{x}$ ray was not lost during the experiment. 
Both of the above mentioned issues can be overcome by alternative fabrication methods, as described in Sec. IV. The observed issues related to the stagnant volume give rise to considerations regarding chip design and the possibility of introducing thin windows of, e.g., silicon nitride. However, the presence of windows may lead to gradual and irreversible irradiation-induced buildup of contaminants on the window surface, whereas any contaminants in the stagnant volume will eventually be removed through diffusion. Also, and especially important for XFEL applications, at sufficiently high $\mathrm{X}$-ray intensities window damage will be inevitable. With a $\mathrm{CBV}$, we achieve a self-healing liquid membrane, which is not permanently damaged and where $\mathrm{x}$-ray diffraction characteristics do not change over time.

\section{CONCLUSIONS AND OUTLOOK}

In the preceding sections, a microfluidic system with an out-of-plane CBV enabling windowless access to a microchannel has been demonstrated. The functionality of the outof-plane CBV has been demonstrated up to driving pressures of 280 mbar corresponding to a burst pressure of $140 \mathrm{mbar}$ (the CBV is placed in the middle of the microfluidic network), and the bursting pressure has been shown to be in good correspondence with the Young-Laplace equation for a channel with circular cross section. From measurements on fluorescently labeled latex beads in water, the flow velocity in the microfluidic channel has been observed to be well described by the Hagen-Poiseuille equation. ${ }^{23}$ One of several possible applications of the investigated microfluidics system is for time-resolved $\mathrm{x}$-ray scattering based on stroboscopic applications of the pump-probe technique. Due to the high beam intensities involved and/or irreversible reactions, sample replacement between successive pump-probe events is often mandatory. For the XFEL sources discussed in Sec. I, the repetition rate covers the range from $10 \mathrm{~Hz}$ to $5 \mathrm{MHz}$. Combining the observed flow rates and access hole diameters, the maximum sample replenishment rate for the system described in this article ranges from $0.3 \mathrm{kHz}(140 \mu \mathrm{m}$ access hole) to $54 \mathrm{kHz}(10 \mu \mathrm{m}$ access hole). In combination with the demonstrated synchrotron beamline feasibility, the windowless microfluidic chip thus presents itself as a candidate sample handling system for several of these new largescale instruments, although reaching the $5 \mathrm{MHz}$ repetition frequency of the Euro-XFEL currently seems unrealistic. Turning to sources already in use today, the $1 \mathrm{kHz}$ repetition rate adopted at several facilities is reached with the presently proposed design using access hole diameters below $90 \mu \mathrm{m}$. However, if a tapered channel is chosen at the windowless access hole diameters above $90 \mu \mathrm{m}$ can be achieved. Regarding time resolution and channel depth, current results from LCLS indicate that time resolutions well down into the tens of femtoseconds should be feasible, mandating correspondingly smaller sample thicknesses as discussed in Sec. I. Within the design paradigm presented in this work, channel depths down to $1 \mu \mathrm{m}$ can be fabricated without any changes to the techniques chosen and without adversely affecting the flow properties of the sample chip.

In situ experiments at ID09b (ESRF) demonstrated that the stagnant volume associated with $\mathrm{CBV}$ region was strongly detrimental to chip performance. Also, significant shadowing effects were observed, complicating data analysis for scattering angles exceeding $2 \theta=12^{\circ}$. These issues could be addressed by replacing the DRIE process with an anisotropic kalium hydroxide $(\mathrm{KOH})$ etch, giving atomically sharp edges with no associated stagnant volume as well as a $34^{\circ}$ slope of the access hole, allowing unimpeded collection of scattered $\mathrm{x}$ rays up to $2 \theta 30^{\circ}$. Initial measurements on $\mathrm{KOH}$-etched prototype devices confirmed the basic feasibility of this approach but at the cost of lowered burst pressure, suggesting a combination of $\mathrm{KOH}$ and DRIE etch for a final design.

An attractive feature of the proposed system is the very low sample consumption, $\mathrm{ml} / \mathrm{h}$, which may be especially relevant for, e.g., protein studies or in case other precious reagents are involved. In combination with straightforward integration of existing lab-on-a-chip technologies, where especially incorporation of optical diagnostics would be a very significant advantage, ${ }^{21}$ the proposed microfluidic sample chip offers interesting new experimental possibilities within time-resolved $x$-ray scattering applications. Furthermore, the windowless design will, together with the vacuum compatibility and the possibility of producing liquid sheets of micrometer thickness, open interesting possibilities for applications in electron scattering and electron microscopy.

\section{ACKNOWLEDGMENTS}

The authors would like to thank B. Vestergaard from the Pharmaceutical Faculty at the University of Copenhagen for assistance with the protein studies as well as H. Lemke from Centre for Molecular Movies and the staff at beamline ID09b at the ESRF for their help with the x-ray measurements. The technical assistance of Keld Theodor from the Niels Bohr Institute in fabricating sample chip holders with incorporated fluid-handling systems is also deeply appreciated. This work was supported by the Danish National Science Foundation's Centre for Molecular Movies and DANSCATT.

${ }^{1}$ T. K. Kim, J. H. Lee, M. Wulff, Q. Kong, and H. Ihee, ChemPhysChem 10, 1958 (2009).

${ }^{2}$ P. Wochner, C. Gutt, T. Autenrieth, T. Dehmer, V. Bugaev, A. D. Ortiz, A. Duri, F. Zontone, G. Grübel, and H. Dosch, Proc. Natl. Acad. Sci. U.S.A. 106, 11511 (2009).

${ }^{3}$ A. Aghababyan et al., XFEL The European X-ray Free-Electron Laser Technical Design Report, HelmHoltz Gemeinschaft, edited by M. Altarelli et al., July 2006.

${ }^{4}$ M. Faubel, B. Steiner, and J. P. Toennies, J. Chem. Phys. 106, 9013 (1997).

${ }^{5}$ B. Winter, E. F. Aziz, N. Ottosson, M. Faubel, N. Kosugi, and I. V. Hertel, J. Am. Chem. Soc. 130, 7130 (2008).

${ }^{6}$ U. Weierstall, R. B. Doak, J. C. H. Spence, D. Stardub, D. Shapiro, P. Kennedy, J. Warner, G. G. Hembree, P. Fromme, and H. N. Chapman, Exp. Fluids 44, 675 (2008)

${ }^{7}$ M. Saes, F. v. Mourik, W. Gawelda, M. Kaiser, M. Chergui, and C. Bressler, Rev. Sci. Instrum. 75, 24 (2004).

${ }^{8}$ M. Cammarata, M. Levantino, F. Schotte, P. A. Anfinrud, F. Ewald, J. Choi, A. Cupane, M. Wulff, and H. Ihee, Nat. Methods 5, 881 (2008).

${ }^{9}$ S. Balslev, A. M. Jorgensen, B. Bilenberg, K. B. Mogensen, D. Snakenborg, O. Geshke, J. P. Kutter, and A. Kristensen, Lab Chip 6, 213 (2006).

${ }^{10}$ T. Lund-Olesen, B. B. Buus, J. G. Howalt, and M. F. Hansen, J. Appl. Phys. 103, 07E902 (2008).

${ }^{11}$ N. Pamme, Lab Chip 7, 1644 (2007).

${ }^{12}$ D. C. Duffy, H. L. Gills, J. Lin, N. F. Sheppard, and G. J. Kellogg, Anal. 
Chem. 71, 4669 (1999).

${ }^{13}$ S. Lai, S. Wang, J. Luo, L. J. Lee, S.-T. Yang, and J. Madou, Anal. Chem. 76, 1832 (2004).

${ }^{14}$ J. V. Zoval and J. Madou, Proc. IEEE 92, 140 (2004).

${ }^{15}$ H. Cho, H.-Y. Kim, J. Y. Kang, and T. S. Kim, J. Colloid Interface Sci. 306, 379 (2007).

${ }^{16}$ R. D. Johnson, I. H. A. Badr, G. Barret, S. Lai, Y. Lu, M. J. Mardou, and L. G. Bachas, Anal. Chem. 73, 3940 (2001).

${ }^{17}$ L. G. Puckett, E. Dikici, S. Lai, M. J. Mardou, L. G. Bachas, and S. Daunert, Anal. Chem. 76, 7263 (2004).

${ }^{18}$ M. Yamada and M. Seki, Anal. Chem. 76, 895 (2004).

${ }^{19}$ F. Laermer and S. Schlip, U.S. Patent No. 5,501,893 (26 March 1996).

${ }^{20}$ F. Persson, L. H. Thamdrup, M. B. L. Mikkelsen, S. E. Jaarlgaard, P. S. Pedersen, H. Bruus, and A. Kristensen, Nanotechnology 18, 245301
(2007).

${ }^{21}$ M. Christensen, K. Haldrup, K. Bechgaard, R. Feidenhans'1, Q. Kong, M. Cammarata, M. L. Russo, M. Wulff, N. Harrit, and M. M. Nielsen, J. Am. Chem. Soc. 131, 502 (2009).

${ }^{22}$ L. Pollack, M. W. Tate, N. C. Darnton, J. B. Knight, S. M. Gruner, W. A. Eaton, and R. H. Austin, Proc. Natl. Acad. Sci. U.S.A. 96, 10115 (1999).

${ }^{23} \mathrm{H}$. Bruus, Theoretical Microfluidics (Oxford University Press Inc., Oxford, 2008).

${ }^{24}$ M. Cammarata, L. Eybert, F. Ewald, W. Reichenback, M. Wulff, P. Anfinrud, F. Schotte, A. Plech, Q. Kong, M. Lorenc, B. Lindenau, J. Raebiger, and S. Polachowski, Rev. Sci. Instrum. 80, 015101 (2009).

${ }^{25}$ K. Haldrup, M. Christensen, M. Cammarata, Q. Kong, M. Wulff, S. O. Mariager, K. Bechgaard, R. Feidenhans'1, N. Harrit, and M. M. Nielsen, Angew. Chem. 48, 4180 (2009). 